\section{La drosophile et l'homme partagent des mécanismes similaires de régulation de la sécrétion d'insuline}

Gérard Manière, Yael Grosjean
Centre des sciences du goût et de l'alimentation, CNRS, INRA, université de Bourgogne Franche-Comté, 6, boulevard Gabriel, F-21000 Dijon, France. yael.grosjean@u-bourgogne.fr
« En mémoire du Pr David $\varepsilon$. Featherstone (University of Illinois at Chicago), scientifique, collègue et ami cher. Nous poursuivrons son inspiration, ses conseils et son travail dans la rigueur, bonne humeur et ouverture d'esprit qui le caractérisaient infailliblement. »

> Se nourrir est indispensable au développement et à la survie de tout individu. Le dérèglement de cette activité peut avoir des conséquences dramatiques sur la santé. Une alimentation excessive entraîne rapidement des problèmes de surpoids et de maladies associées [1]. À l'inverse, une prise alimentaire trop faible est également préjudiciable [2]. La compréhension des phénomènes biologiques qui gouvernent la prise alimentaire est ainsi un enjeu très important de santé publique. Dans ce contexte, de nombreuses équipes de recherche cherchent à en percer les secrets en utilisant notamment des modèles animaux.

Les animaux trouvent dans leur alimentation les nutriments nécessaires pour couvrir tous les besoins nutritionnels indispensables au fonctionnement des cellules, à leur survie et à leur croissance. Cette prise alimentaire va déclencher la synthèse et la libération de nombreuses hormones pour réguler le métabolisme général de l'organisme. Ainsi, chez les mammifères, une hausse de la concentration en glucose sanguin après un repas riche en sucre provoque une libération d'insuline par les cellules $B$ du pancréas pour réguler finement la glycémie. La sécrétion d'insuline est aussi induite par un repas riche en protéines. Ces protéines sont constituées d'un enchaînement d'acides aminés. Un de ces acides aminés, la leucine, non synthétisable par l'organisme et issue de la digestion du bol alimentaire, est bien connue pour déclencher la libération d'insuline au niveau des cellules $\beta$ du pancréas [3]. La leucine pourrait provoquer cette libération d'insuline par l'intermédiaire d'un transporteur d'acides aminés de type LAT-I ( $L$-amino acid transporter-1) présent dans ces cellules $B$ du pancréas [4].

Chez la larve de drosophile, les principaux DILP (Dro-

$(\rightarrow)$ Voir la Synthèse de S. Raisin et al., $\mathrm{m} / \mathrm{s} \mathrm{n}^{\circ} 5$, mai 2002 , sophila insulin-like page 610 peptides) [5] $(\rightarrow)$,

des peptides apparentés à l'insuline et aux IGF (insulin growth factors), sont synthétisés notamment par un groupe de quatorze cellules, localisées dans le cerveau. Ces cellules sont considérées comme les homologues des cellules $B$ du pancréas des mammifères. Ces DILP sont impliqués, comme chez les mammifères, dans la régulation du métabolisme des sucres, des lipides et de la prise alimentaire [6]. Toutefois, chez la drosophile, la régulation de la sécrétion des DILP par les nutriments issus du bol alimentaire était décrite jusqu'à maintenant comme indirecte. En effet, le modèle actuel de libération des DILP par les cellules du cerveau repose sur la mise en jeu obligatoire d'hormones produites par différents organes (tissu adipeux, tube digestif, etc.) $[6,7]$.

Au fil de nos recherches, nous avons fortuitement identifié la présence d'un transporteur de leucine appelé Minidiscs dans les cellules sécrétrices de DILP du cerveau des drosophiles. Nous avons ainsi testé l'hypothèse que ces cellules neurosécrétrices de DILP seraient capables de détecter directement les nutriments comme la leucine [8]. Cette information est particulièrement importante car la drosophile est un modèle puissant pour déterminer les facteurs génétiques qui gouvernent des fonctions physiologiques universelles, telles que la prise alimentaire et la libération d'insuline. L'accès au génome de cette petite mouche est en effet précis et aisé. De plus, une quantité toujours plus sophistiquée d'outils génétiques est développée régulièrement par la communauté scientifique, ce qui en fait l'un des meilleurs modèles biologiques.

Nous avons ainsi montré [8], avec les Drs Ziegler, Geillon et Featherstone, que suite à un repas, la leucine va agir sur les cellules spécialisées dans la synthèse des DILP du cerveau des larves de drosophile pour déclencher leur libération dans I'organisme. Ces DILP vont ensuite réguler la glycémie et permettre une bonne croissance. Nous avons également mis en évidence que cet événement nécessite la mobilisation d'acteurs moléculaires indispensables. Le premier est la protéine Minidiscs. II s'agit d'un transporteur de la leucine qui relaie son action au sein des cellules sécrétrices de l'insuline. Le second est la glutamate déshydrogénase, une enzyme qui permet de déclencher la libération d'insuline (Figure 1) [8].

Ces résultats sont d'une grande importance car ils mettent en évidence une voie de signalisation originale qui pour- 


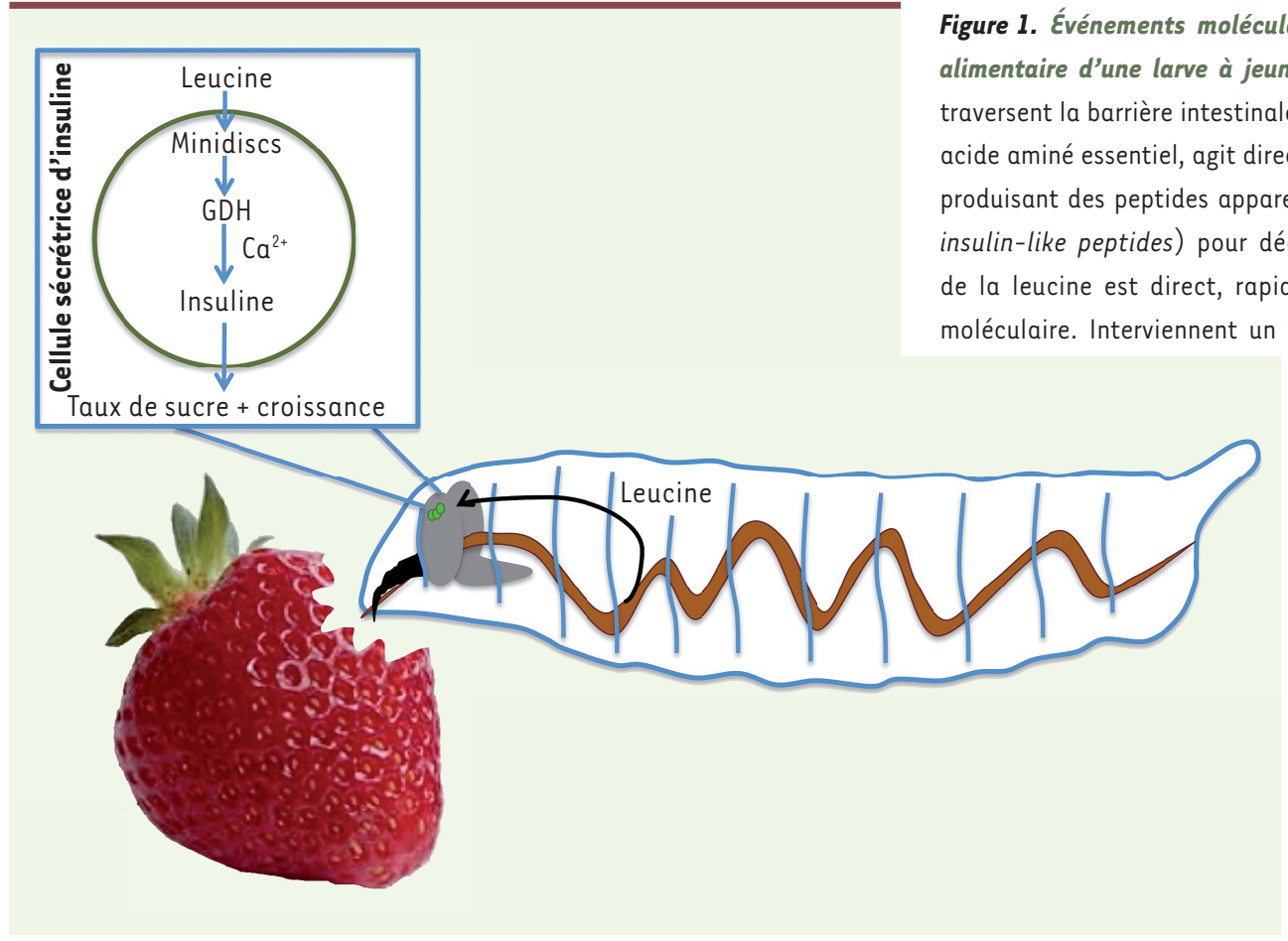

Figure 1. Événements moléculaires mis en jeu lors de la prise alimentaire d'une larve à jeun. Après digestion, les nutriments traversent la barrière intestinale. Parmi ces nutriments, la leucine, git directement sur des cellules cérébrales apparentés à l'insuline (DILP, Drosophila pour déclencher leur sécrétion. Cet effet mobilise alors une cascade discs) et une enzyme (la glutamate déshydrogénase, GDH) qui entraînent une augmentation locale de $\mathrm{Ca}^{2+}$ responsable de la libération des DILP dans l'organisme. La dérégulation de ces acteurs moléculaires entraîne une modification de la concentration en sucre (le tréhalose) circulant dans l'organisme larvaire et a par conséquent une incidence sur la croissance des larves.

rait être grandement conservée dans l'ensemble du règne animal, contrairement à ce qui était pensé jusqu'à présent.

Cette avancée, par la compréhension fine des processus biologiques déterminant la prise alimentaire, permet d'envisager de spectaculaires perspectives. II sera par exemple très intéressant de déterminer si les mammifères possèdent des cellules sécrétrices d'insuline localisées dans leur cerveau qui mobiliseraient des voies de signalisation équivalentes, et notamment les transporteurs d'acides aminés LAT-1. Les travaux réalisés sur la drosophile et leur éventuelle confirmation chez l'homme ouvrent ainsi des pistes passionnantes pour envisager de nouvelles stratégies de lutte contre les dysfonctionnements liés à l'alimentation. $\diamond$

Drosophila and humans share similar mechanisms of insulin secretion

\section{LIENS D'INTÉRÊT}

Les auteurs déclarent n'avoir aucun lien d'intérêt concernant les données publiées dans cet article.

\section{REMERCIEMENTS}

Les auteurs remercient l'ANR (ANR-JCJC, GGCB2010) et la Commission Européenne (ERC Starting Grant, GliSFCo-311403) pour leur soutien financier.

\section{RÉFÉRENCES}

1. Van Baak MA. Nutrition as a link between obesity and cardiovascular disease: how can we stop the obesity epidemic? Thromb Haemost 2013 ; 110 : 689-96.
2. Martins VJ, Toledo Florêncio TM, et al. Long-lasting effects of undernutrition. Int J Environ Res Public Health $2011 ; 8: 1817-46$.

3. Sener A, Somers G, Devis G, Malaisse WJ. The stimulussecretion coupling of amino acid-induced insulin release. Biosynthetic and secretory responses of rat pancreatic islet to L-leucine and L-glutamine. Diabetologia $1981 ; 21: 135-42$.

4. Cheng Q, Diez Beltran V, Chan SM, et al. System-L amino acid transporters play a key role in pancreatic beta-cell signalling and function. J Mol Endocrinol $2016 ; 56: 175-87$.

5. Raisin S, Pantalacci S, Léopold P. Les insulines des invertébrés sèment le trouble. Med Sci (Paris) 2002 ; $18: 610-5$.

6. Rajan A, Perrimon N. Of flies and men : insights on organismal metabolism from fruit flies. BMC Biology $2013 ; 11: 38$.

7. Andersen DS, Colombani, Leopold P. Coordination of organ growth : principles and outstanding questions from the world of insects. Trends Cell Biol 2013;23 : 336-44.

8. Manière G, Ziegler AB, Geillon F, et al. Direct sensing of nutrients via a LAT-1 transporter in Drosophila insulinproducing cells. Cell Reports $2016 ; 17: 137-48$.

\section{LA FONDATION PREMUP : UN OPÉRATEUR DE TERRAIN EN PÉRINATALITÉ RECONNU POUR SON EXCELLENCE ET SON INTERDISCIPLINARITÉ} La Fondation de coopération scientifique PremUp, unique en Europe, intervient sur la prévention FONDATION DE COOPÉRATION SCIENTIFIQUEE
SUR LA GROSSESSE ET LA PRÉMATURIÉ du handicap à la naissance, par la protection de la santé de la femme enceinte et du nouveau-né.

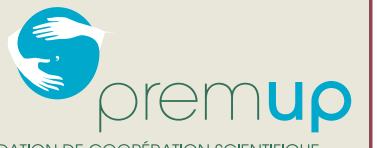

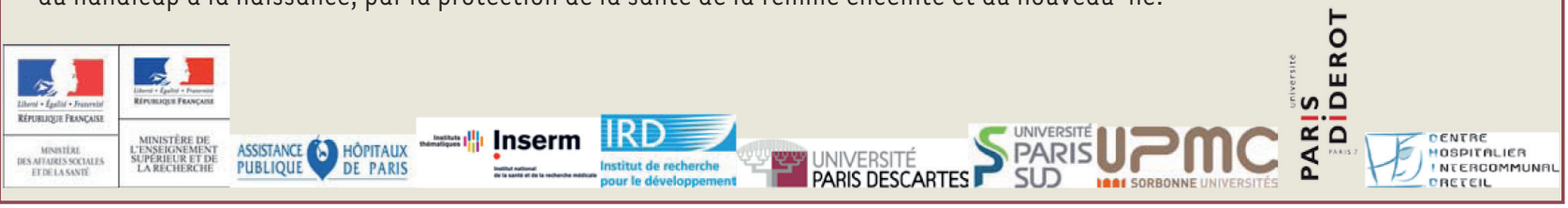

